\title{
How to Develop the Medical Neighborhood
}

\author{
Joseph D. Feuerstein $^{1}$ - Victoria Sheppard ${ }^{1}$ Adam S. Cheifetz ${ }^{1}$. \\ Kim Ariyabuddhiphongs ${ }^{2}$
}

Received: 3 May 2016 / Accepted: 14 July 2016 / Published online: 22 July 2016

(C) Springer Science+Business Media New York 2016

\begin{abstract}
Significant attention has been directed towards developing the medical home and improving the patient experience. The medical home is targeted towards optimizing the quality of patient care while also reducing overall costs. An extension of the medical home is the concept of a medical neighborhood. The medical neighborhood utilizes the success of the medical home and incorporates it into the coordination of care between primary care physician and specialists. In order to create an ideal system, though, the framework for making referrals, ordering tests prior to referrals, documentation and communication of recommendations must be addressed a priori. In this perspective we discuss the necessary steps to implement a medical neighborhood for patients with chronic medical conditions and the use of medical technology to facilitate this process.
\end{abstract}

This article is part of the Topical Collection on Systems-Level Quality Improvement

Joseph D. Feuerstein

jfeuerst@bidmc.harvard.edu

Victoria Sheppard

Vsheppa@bidmc.harvard.edu

Adam S. Cheifetz

acheifet@bidmc.harvard.edu

Kim Ariyabuddhiphongs

kariyabu@bidmc.harvard.edu

1 Department of Medicine and Division of Gastroenterology, Beth Israel Deaconess Medical Center, Harvard Medical School, 110 Francis Street 8E Gastroenterology, Boston, MA 02215, USA

2 Department of Medicine, Beth Israel Deaconess Medical Center, Harvard Medical School, Boston, USA

\section{Introduction}

Significant attention has been directed to developing the medical home and improving the patient experience. The medical home is targeted towards optimizing the quality of patient care while also reducing overall costs $[1,2]$. However, some models of the medical home do not specifically incorporate the specialists under the same roof [3]. Often, space limitations and availability limits this geographic co-location. To continue to enhance the care of patients, the medical home must cross walls and transform into a medical neighborhood that includes specialists regardless of their physical location [4]. Patients with chronic medical disease require close coordination of care between primary care physicians (PCP) and specialists [4]. In order to create an ideal system, though, the framework for making referrals, ordering tests prior to referrals, documentation and communication of recommendations must be addressed. In this commentary we discuss the necessary steps to implement a medical neighborhood and the results from a recent focus group of specialists (cardiologists, endocrinologists, pulmonologists, and gastroenterologists) and primary care physicians we performed on this topic.

\section{Importance of the medical neighborhood}

As medical expenditures rise, the goal to control cost while providing high quality care is of increasing importance. The concept of the medical home focuses on controlling cost while providing quality based care. However, primary care expenditures only account for $6 \%$ of total healthcare spending in the United States [5]. To better control costs, specialty care needs to be optimized to provide high quality care at acceptable healthcare costs. Ideally, this can be achieved through the development of a medical neighborhood. The goals of the 
medical neighborhood are to improve patient safety, experience, quality evidence based care, while also reducing cost and unnecessary duplication of services. To coordinate this care, however, is no simple task. A survey of primary care physicians reported that a primary care physician had 229 other physicians working in 117 practices [5]. Given how fragmented care is between specialists and PCPs, provided quality care can be challenging. Many chronic diseases (e.g. heart failure, myocardial infarction, asthma, diabetes, inflammatory bowel disease etc.) have performance measures which are used to assess the overall quality of care delivered [6]. Often, close collaboration between specialists and PCPs are necessary to comply with these measures. For example, some specialty clinics may not offer vaccinations for influenza and pneumococcal infection which are required in patients with asthma, diabetes, and inflammatory bowel disease. Similarly, if PCPs utilize colonoscopy to achieve compliance with colon cancer screening quality measures, a clear collaboration with the gastroenterologist is necessary to ensure that any patient who does not keep their appointment or does not achieve an adequate screening colonoscopy is sought out for ongoing screening [6]. Without this bidirectional communication, both the PCP and specialist may inadvertently fail to meet necessary performance measures. These performance measures are being used by insurance companies and government agencies to both rate clinicians and practices on quality of care, as well as establish reimbursements based on the documented quality of care provided [7, 8]. Recently, a gastroenterology group from Illinois utilized an expanded medical home model starting in 2014 and successfully lowered cost by $10 \%$ and reduced hospitalization payments by $57 \%$ [9]. Achieving these savings was through a multidisciplinary approach to the management of a chronic disease [9]. Ultimately, to comply with the reportable performance measures, PCPs and specialists need to develop more structured alliances that streamline care and control costs. The goal of the medical neighborhood is to lay the groundwork for this co-management scheme of patients with chronic diseases between PCPs and specialists. Streamlining the use of electronic medical records can dramatically enhance the integration of the medical neighborhood between PCPs and specialists.

\section{Barriers to care}

\section{Referral}

Barriers to care start with inadequate information being provided at the time of referral to a specialist. A clear and succinct question is necessary when referring patients to consultants. Along with the clinical question, primary care physicians (PCP) should indicate if they would like a one-time consultation, to undertake co-management of the patient's issue(s), or for specialist to completely take over care for the specific condition. Additionally, all relevant medical records should be sent to the consultant prior to the visit. While many medical centers utilize electronic records, private and community practices are not always integrated. Inadequate historical records may lead to inappropriate testing and incomplete recommendations resulting in increased medical costs. In addition, the patient and specialist may feel that their time is not being effectively utilized. To obviate this issue, when formulating the relationship, specialists and PCPs should standardize the way referrals are placed and what records are necessary prior to or at the time of consultation [3].

\section{Ordering tests prior to referral}

The ordering of tests prior to referring patients has the potential to reduce costs but if done inappropriately it could unnecessarily increase expenditure. Prior to a referral for chest pain to a cardiologist, obtaining a stress test may reduce the number of specialty visits needed and may provide the cardiologist with additional important information to augment the initial visit. On the other hand, if a stress test, however, is not necessary then inappropriate testing raises costs with no added quality of care provided. In contrast, obtaining baseline hemoglobin a1c, creatinine, and urinalysis prior to an endocrinology referral for diabetes may be extremely helpful. However, if both the specialist and the referring PCP duplicate the ordering than any cost-savings is lost. High value care is achieved when only the appropriate tests are ordered and unnecessary testing is avoided. Improved communication between specialists and PCPs is necessary to help standardize and improve the quality of care of patients prior to their initial visit with the consultant. Reducing the need for even one specialty visit with adequate pre-visit testing can lead to significant cost-savings. Electronic ordering templates can be designed by specialties to better streamline this process and provide decision support tools to the clinician ordering the test prior to the referral.

\section{Documentation and recommendations}

In addition to summarizing the patient's medical history, issues, and plans to evaluate/treat these problems, the medical note is also utilized for billing purposes and medico-legal documentation. All too often, the note is inundated with information unrelated to the referral question, is difficult to read quickly, and recommendations are hard to find. As a result, in a focus group of specialists and PCPs at our institution many commented that the medical note is no longer a means of communication but rather is primarily utilized for the purposes of medico-legal documentation and billing. In fact, many of these physicians (PCPs and specialists) commented that they do not expect anyone to actually read the note. 
A potentially more concise section of the note with the key recommendations is the assessment and plan. Ideally, the assessment should succinctly summarize the referral question along with the differential diagnosis and clearly state the planned evaluation and treatment. Importantly, whoever is responsible for carrying out the action plan from these recommendations is often missing. As an example, patients with inflammatory bowel disease should be vaccinated for pneumonia and influenza. While some gastroenterology practices may have these vaccinations available and perform them in their office, other subspecialty groups lack the means to vaccinate patients. When these physicians recommend immunizations it may not be clear to the primary care practitioner that the gastroenterologist is directing the referring provider to give the vaccine. Similarly, when incidental findings are identified during the specialist visit, such as a pulmonary nodule necessitating repeat imaging, clear communication back to the primary care physician indicating who will be responsible for ordering and following up the test is necessary for adequate closed-loop communication. The use of email within an electronic record system can provide an easy means of closedloop communication as well as medico-legal documentation.

\section{Communications between specialists and primary care physicians}

In order to ensure optimal and safe patient care, adequate communication of findings and results must readily flow between specialists and PCPs. During our focus group discussion, many physicians expressed concern that the current electronic medical documentation fails to provide a consistent means of communication between physicians. While some physicians electronically forward notes within the electronic medical record system, several PCPs at our meeting noted that this is not adequate. They mentioned that a busy primary care physician is inundated with forwarded notes and feel it is difficult, if not impossible, to stay abreast of all these notes given their length and lack of standardization. These notes are often not reviewed until the time of the patient's next visit. Several of the specialists pointed out that if information contained in these notes is of critical importance, they would also utilize email to notify the PCP. This decision to use email is predicated on the fact that they feel the medical documentation they authored was not adequate closed-loop communication. Somewhat surprisingly, few physicians reported calling the referring physician to discuss the issue over the phone, or setting up a face-to-face meeting.

While duplicating communication (e.g. documentation in a medical note and email) is not ideal, the current system does not suffice. In order for a medical neighborhood to succeed when practices are not within the confines of the same office, appropriate means of communication is critical. Just like the medical home utilizes team based care to reduce the burden on the primary care, a medical neighborhood requires a similar system to improve the overall flow of communication. Electronic record systems that are integrated typically allow for internal communication which serves as both communication and documentation of the visit.

\section{Novel concepts for developing a medical neighborhood}

Given the challenges mentioned above, novel concepts are needed to establish an effective medical neighborhood. Ideally, in the current design of the medical system, establishing a patient navigator in both the primary care clinics and specialty clinics could serve to help standardize the care and insure that communication flows seamlessly between both clinics. Unfortunately, however, this additional cost is unlikely to be feasible in many clinics. While the use of standardized electronic medical record communication is attractive to meet the goals of a medical neighborhood, our panel unanimously felt that this was not being utilized as an effective means of communication between providers.

Two concepts that were proposed as means to utilized technology to achieve the goals of the medical neighborhood. One concept focused on a low cost use of videoconferencing between specialists and providers. What seems to be lacking in most systems is a standardized means of communication between providers. To that end, a video conference call can be performed weekly between specialists and PCPs. At that time, all patients that are co-managed would be reviewed and recommendations would be discussed. Such a process would also facilitate compliance with disease specific quality measures as well. This process would add minimal costs. The resources for telecommunication and video conferencing are available at most practices and if unavailable are readily available free or for nominal charges online. This concept would also be feasible among smaller primary care group practices so long as they standardize where referrals are sent. By standardizing this communication into a set conference, this would allow for better discussion of any patients in need of additional care and would prevent recommendations from being missed in the electronic record system. This would then obviate the need for additional emails or phone calls between provides at unscheduled times unless a true medical emergency was present. A significant limitation of this intervention is the additional documentation that will be required of the communication between the specialists and PCP and then subsequent follow up calls and/or visits with patients to accomplish these tasks.

A different method which works to avoid the additional documentation and additional patient visits is the integration of recommendations and quality measures into the electronic record system. In our EHR, we utilized inflammatory bowel disease as a pilot program to develop a screen where all disease parameters could be summarized and all necessary quality metrics could be viewed at once (Figs. 1 and 2). This tab 


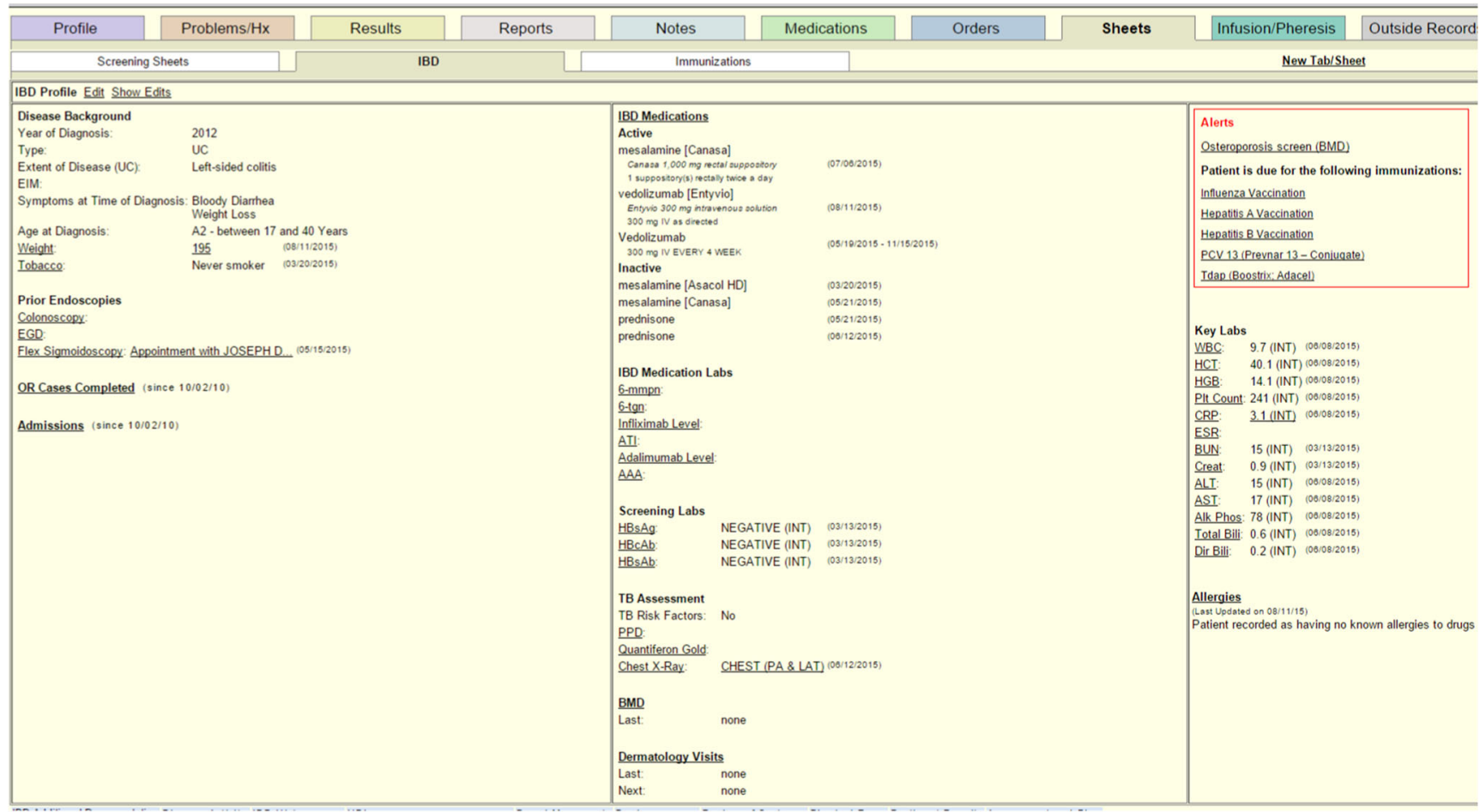

Fig. 1 EHR screenshot template of integrated IBD worksheet

\begin{tabular}{|c|c|c|c|c|c|c|c|c|c|}
\hline $\begin{array}{l}\text { IBD Additional } \\
\text { Documentation }\end{array}$ & $\begin{array}{l}\text { Disease } \\
\text { Activity }\end{array}$ & IBD History & $\underline{\mathrm{HPI}}$ & $\frac{\text { Bowel }}{\text { Movements }}$ & $\underline{\text { Denies }}$ & $\frac{\text { Review of }}{\text { Systems }}$ & $\frac{\text { Physical }}{\text { Exam }}$ & $\frac{\text { Pertinent }}{\text { Results }}$ & $\begin{array}{l}\text { Assessment } \\
\text { and Plan }\end{array}$ \\
\hline \multirow[t]{3}{*}{ IBD Labs 1} & WBC & $\underline{\mathrm{HCT}}$ & HGB & MCV & Plt Count & BUN & Creat & ESR & $\underline{\mathrm{CRP}}$ \\
\hline & $05 / 12 / 2014$ & $05 / 12 / 2014$ & $05 / 12 / 2014$ & $05 / 12 / 2014$ & $05 / 12 / 2014$ & $05 / 12 / 2014$ & $05 / 12 / 2014$ & $12 / 08 / 2008$ & $12 / 08 / 2008$ \\
\hline & 5.7 (INT) & 40.6 (INT) & 13.7 (INT) & $100^{*}$ (INT) & 191 (INT) & 14 (INT) & 0.6 (INT) & 4 (INT) & 0.4 (INT) \\
\hline \multirow[t]{3}{*}{ IBD Labs 2} & $\underline{A l b}$ & Total Bili & Dir Bili & $\underline{\mathrm{ALT}}$ & AST & Alk Phos & Tot Prot & & \\
\hline & $12 / 14 / 2009$ & $05 / 12 / 2014$ & 09/07/2011 & $05 / 12 / 2014$ & $05 / 12 / 2014$ & $05 / 12 / 2014$ & & & \\
\hline & 4.6 (INT) & 0.4 (INT) & 0.1 (INT) & $18(\mathrm{INT})$ & $23(\mathrm{INT})$ & 40 (INT) & & & \\
\hline \multirow[t]{3}{*}{ BBD Labs 3} & Iron & Ferritin & $\underline{\mathrm{B} 12}$ & Folate & $\underline{\text { TTG-IGA }}$ & $\lg A$ & & & \\
\hline & & & $05 / 12 / 2014$ & $05 / 12 / 2014$ & & & & & \\
\hline & & & 512 (INT) & $\frac{\text { GREATER }}{\text { TH (INT) }}$ & & & & & \\
\hline \multirow[t]{3}{*}{ IBD Labs 4} & $\underline{\mathrm{HAV} A \mathrm{Ab}}$ & $\underline{H B s A b}$ & HBsAg & $\underline{\mathrm{HBCAb}}$ & $\underline{\mathrm{HCV}} \mathrm{AB}$ & $\begin{array}{l}\text { Quantiferon } \\
\underline{\text { Gold }}\end{array}$ & $\underline{P P D}$ & & \\
\hline & & $06 / 14 / 2010$ & $06 / 14 / 2010$ & $06 / 14 / 2010$ & $06 / 14 / 2010$ & & & & \\
\hline & & $\begin{array}{l}\text { NE GATIVE } \\
\text { (INT) }\end{array}$ & $\begin{array}{l}\text { NE GATIVE } \\
\text { (INT) }\end{array}$ & $\begin{array}{l}\text { NE GATIVE } \\
\text { (INT) }\end{array}$ & NEGATIVE (INT) & & & & \\
\hline IBD Labs 5 & TPMT & 6-tgn & $\underline{6-m m p n}$ & $\begin{array}{l}\text { Infliximab } \\
\underline{\text { Level }}\end{array}$ & ATI & $\begin{array}{l}\text { Adalimumab } \\
\text { Level }\end{array}$ & AAA & & \\
\hline \multirow[t]{3}{*}{ IBD Imaging } & MRE & $\frac{\text { CT ABD \& }}{\text { Pelvis }}$ & $\begin{array}{l}\text { UG| Air w/ } \\
\underline{\underline{\text { SBFT }}}\end{array}$ & MR Pelvis & Osteop. Screen & Chest X-Ray & & & \\
\hline & & & $09 / 21 / 1995$ & & $12 / 04 / 2009$ & & & & \\
\hline & & & $\begin{array}{l}\text { UGI/SMALL } \\
\text { BOWEL } \\
\text { (BARIUM) }\end{array}$ & & $\begin{array}{l}1101 \text { BONE } \\
\text { DENSITOMETRY }\end{array}$ & & & & \\
\hline \multirow[t]{3}{*}{$\frac{\text { IBD }}{\text { Procedures }}$} & Colonoscopy & $\underline{E G D}$ & $\begin{array}{l}\text { Flex } \\
\underline{\text { Sigmoidoscopy }}\end{array}$ & & & & & & \\
\hline & $02 / 25 / 2013$ & & & & & & & & \\
\hline & $\begin{array}{l}\text { Tissue: GI } \\
\text { BX (9 JARS) }\end{array}$ & & & & & & & & \\
\hline IBD Pathology & $\frac{\text { Gl Mucosal }}{\mathrm{BX}}$ & Upper GI BX & Lower GI BX & & & & & & \\
\hline
\end{tabular}

Fig. 2 Screenshot of lab summary relevant to IBD on single screen IBD worksheet 
IBD Profile

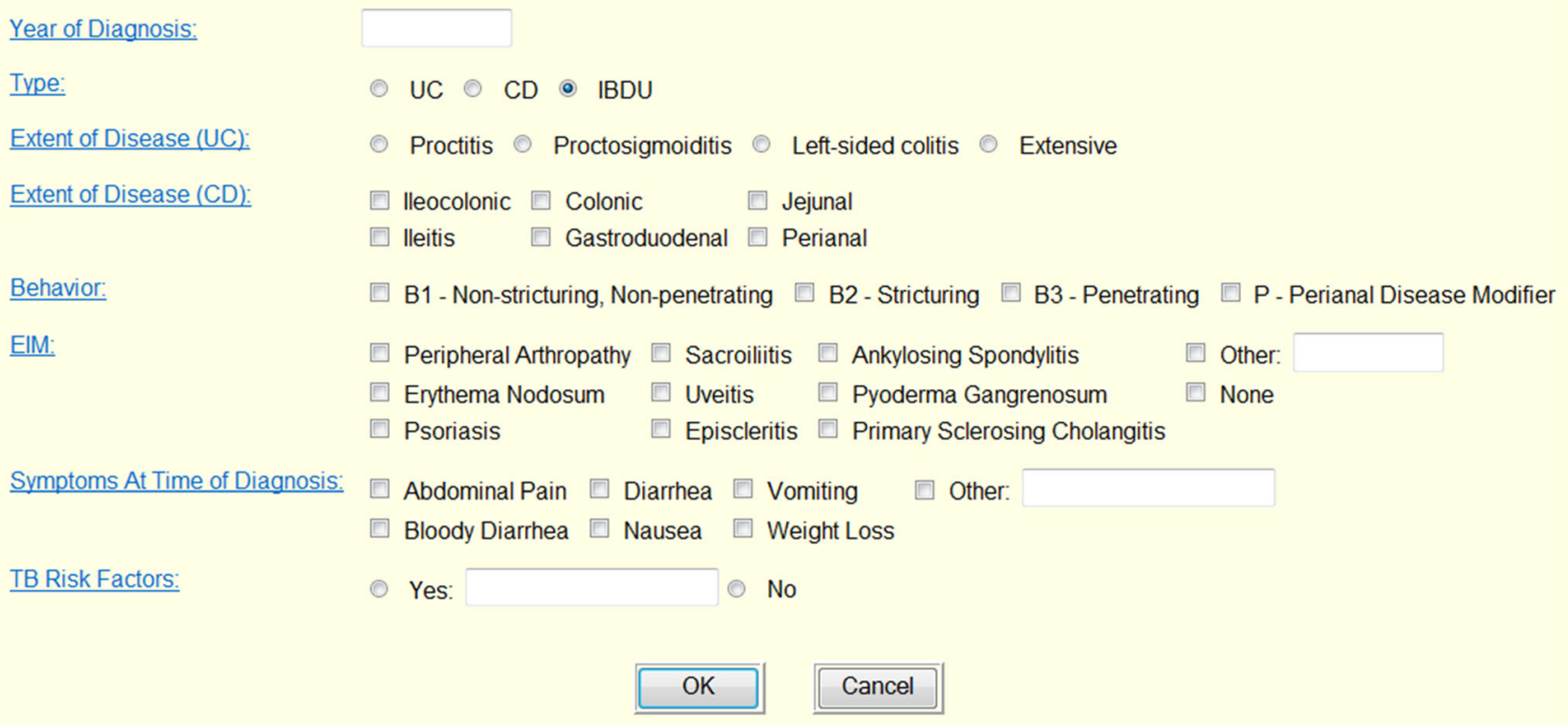

Fig. 3 Standardization of input into IBD worksheet

allowed for the documentation of initial history and summarized all key quality measures in IBD. To avoid errors, the input categories into the worksheet are all standardized with checkbox categories (Fig. 3). Also, the tab is automatically updated with new test results and has alerts built into it when items are out of compliance. This innovation would then allow for the monitoring of compliance with necessary quality metrics and provide alerts to the PCP and specialists for any measures that are out of compliance. Additionally, this technology automatically alerts providers to any measure that is due to be repeated or reassessed within 30 days. The goal of this tab was to provide a single screen that served as the means of monitoring compliance with quality measures and could be utilized by both specialists and PCPs to monitor compliance. This system also allowed for the data on compliance to be exported and actively monitored. The ability to not just comply with measures but also export them is critical. Many of these quality measures are required to adhere to PQRS requirements and insurance companies are increasingly likely to require proof of compliance to demonstrate the quality of care being provided. A system similar to the one used in our EHR can be developed in other systems with proper IT support and physician design. Likewise, this concept can be extrapolated to other diseases with quality metrics and issues that are co-managed between PCPs and specialists.

\section{Conclusion}

The medical neighborhood is the framework by which PCPs and specialists can continue to provide high quality and cost- effective care to patients despite being in different physical locations. The American College of Physicians has developed frameworks to develop these relationships [3]. There are multiple key building blocks that must be established at the onset of developing a medical neighborhood. Practices need to start brainstorming to develop their ideal collaboration model between PCPs and specialists. The first step in any medical neighborhood is always standardizing the means for communication about referrals and subsequent recommendations. Relying on the medical record alone may not be adequate and may result in inadvertent lapses in care. However, if notes become standardized with clear recommendations and assignments of responsibility, this may result in improved compliance of reportable performance measures in chronic disease. Our focus group postulated that either using a standardized video conference between specialists and PCPs or using the EHR to provide a single screen were all the disease specific information and quality measures are summarized would ultimately enhance communication between PCPs and specialists and compliance with quality measures. A medical neighborhood can serve to enhance the overall quality of care, but significant work is necessary to develop the framework of this new model of care $[1,3,4]$. There is no single model that will fit every practice, but at this time, practices should begin brainstorming on how this new co-management of chronic disease will be established.

\section{Compliance with Ethical Standard}

Conflict of Interest No authors report any relevant conflicts of interest. 


\section{References}

1. Schwenk, T.L., The patient-centered medical home: one size does not fit all. JAMA. 311(8):802-803, 2014.

2. Nelson, K.M., Helfrich, C., Sun, H., et al., Implementation of the patient-centered medical home in the veterans health administration: Associations with patient satisfaction, quality of care, staff burnout, and hospital and emergency department use. JAMA Internal Medicine. 174(8):1350-1358, 2014.

3. Kirschner, N., and Greenlee, M., The patient-centered medical home neighbor: the interface of the patient-centered medical home with specialty/subspecialty practices. A Position Paper of the American College of Physicians. American College of Physicians, Philadelphia, PA, 2010.

4. Huang, X., and Rosenthal, M.B., Transforming specialty practicethe patient-centered medical neighborhood. New England Journal of Medicine. 370(15):1376-1379, 2014.

5. Pham, H.H., O'Malley, A.S., Bach, P.B., Saiontz-Martinez, C., and Schrag, D., Primary care Physicians' links to other physicians through Medicare patients: the scope of care coordination. Annals of internal medicine. 150(4):236-242, 2009.

6. US Department of Health and Human Services. HRSA Health Center Program Quality of Care. http://bphc.hrsa. gov/qualityimprovement/performancemeasures/qualitycare.html. Accessed march 23, 2016.

7. Center for Medicare and Medicaid Service. Value-Based Payment Modifier. https://www.cms.gov/medicare/medicare-fee-for-servicepayment/physicianfeedbackprogram/valuebasedpaymentmodifier. html\#What is the Value-Based Payment Modifier (Value Modifier) Accessed March 23, 2016.

8. VanLare, J.M., and Conway, P.H., Value-based purchasing National Programs to Move from Volume to Value. New England Journal of Medicine. 367(4):292-295, 2012.

9. Dyrda L. Illinois Gastroenterology Group's Crohn's medical home shows $10 \%$ cost reduction -7 things to know. http://www. beckersasc.com/gastroenterology-and-endoscopy/illinoisgastroenterology-group-s-crohn-s-medical-home-shows-10-costreduction-7-things-to-know.html. Accessed March 23, 2016. 